\title{
Percentage Body Fat by Dual-Energy X-Ray Absorptiometry Is Associated With Menstrual Recovery in Adolescents With Anorexia Nervosa
}

\author{
Sarah Pitts, M.D. ${ }^{a,}$, Emily Blood, Ph.D. ${ }^{b}$, Amy Divasta, M.D., M.Sc. ${ }^{c}$, and Catherine M. \\ Gordon, M.D., M.Sc. ${ }^{d}$
}

aDivision of Adolescent/Young Adult Medicine and Division of Endocrinology, Boston Children's Hospital, Boston, Massachusetts ${ }^{b}$ Clinical Research Center and Division of Adolescent/Young Adult Medicine, Boston Children's Hospital, Boston, Massachusetts 'Division of Adolescent/ Young Adult Medicine and Division of Gynecology, Boston Children's Hospital, Boston, Massachusetts dDivision of Adolescent Medicine, Hasbro Children's Hospital, Providence, Rhode Island

\begin{abstract}
Purpose-To evaluate mediators of resumption of menses (ROM) in adolescents with anorexia nervosa (AN).

Methods-Anthropometrics, body composition by dual-energy X-ray absorptiometry, hormonal studies, and responses to mental health screens were obtained at 6-month intervals for 18 months in 37 adolescents with AN randomized to the placebo arm of a double-blind treatment trial. Outcomes were compared between subjects with menstrual recovery and those without.

Results-Twenty-four subjects (65\%) had ROM. Higher percentage body fat was associated with ROM (odds ratio, 1.19; $95 \%$ confidence interval, 1.06, 1.33; $p<.01$ ), as was body mass index and percent median body weight. Estradiol $\geq 30 \mathrm{ng} / \mathrm{mL}$ alone did not predict menses $(p=$. 08) but was associated with ROM when coupled with percent mean body weight (odds ratio, 2.49; 95\% confidence interval, 1.09, 5.65; $p=.03$ ). Changes in leptin, cortisol, and mental health were not associated with return of menses.
\end{abstract}

Conclusions-Percentage body fat may be an additional, useful clinical assessment to follow in caring for adolescents with AN.

\section{Keywords}

Adolescent; Anorexia nervosa; Amenorrhea; Body composition; Leptin

\footnotetext{
(C) 2014 Society for Adolescent Health and Medicine. All rights reserved.

*Address correspondence to: Sarah Pitts, M.D., Boston Children's Hospital, Adolescent Medicine, 00 Longwood Avenue, Boston, MA 02115. sarah.pitts@ childrens.harvard.edu (S. Pitts).

IMPLICATIONS AND CONTRIBUTION: In addition to change in body mass index (BMI), percentage body fat may be a useful clinical measure to inform return of menses in adolescents recovering from anorexia nervosa (AN).

Dr. Amy Divasta and Dr. Catherine M. Gordon are co-senior authors.
} 
Amenorrhea is commonly seen in adolescents with AN. Within 3-6 months of achieving a BMI between the 14th and 39th percentiles, many adolescents with AN have resumption of menses (ROM). Additionally, an estradiol level $>30 \mathrm{pg} / \mathrm{mL}$ is predictive of ROM [1]. Leptin may also play a neuroendocrine role in $\mathrm{AN}[2,3]$. After leptin supplementation, women with hypothalamic amenorrhea exhibited ROM [4]. Since leptin reflects fat mass, body composition may correlate with ROM. Psychopathology and stress hormones have also been implicated as mediators of menstrual dysfunction [5].

Our current analysis aimed to compare previously established weight and hormonal goals [1] to contemporary adolescents with AN and to evaluate other correlates of ROM.

\section{Methods}

\section{Participants}

Thirty-seven females, aged 13-27 years, diagnosed with AN by their outpatient medical providers and randomized to the placebo arm of a clinical trial completed the baseline visit; 29 completed the 18-month visit [6]. The Institutional Review Board at Boston Children's Hospital approved the study.

\section{Study assessments}

Study visits occurred at baseline, 3, 6, 12, and 18 months. Body composition by dual-energy X-ray absorptiometry (DXA), serum estradiol (high-performance liquid chromatography tandem mass spectrometry, Esoterix, Calabasas Hills, CA), and serum cortisol (electrochemiluminescent immunoassay, Roche Cobas, Mannheim, Germany) were obtained at baseline and at every 6 months. Serum leptin (radioimmunoassay, Millipore, St. Charles, MO) was measured at baseline $(\mathrm{n}=11)$ and at 18 months $(\mathrm{n}=10)$. The Beck Depression Inventory-I [7] and 26-item Eating Attitudes Test [8] were completed at baseline, 6-, 12-, and $18-$ month visits.

\section{Statistical analysis}

Descriptive statistics were used to characterize the study subjects. Fisher's exact test and $t$ test compared baseline characteristics of those who did and did not achieve ROM. Longitudinal logistic regressions via generalized estimating equations were performed to test the association of time-varying clinical and mental health variables with menses over time. These models used all available observations over time from all participants. Both change from baseline and value at the time of visit were tested as predictors in the separate logistic models. Models were adjusted for time of visit (which was significantly associated with ROM) to account for treatment effect. Significance was considered a two-sided p value $<.05$.

\section{Results}

Few baseline differences existed between subjects with ROM $(\mathrm{n}=24,65 \%)$ and those without $(\mathrm{n}=13$; Table 1$)$. Subjects were primarily Caucasian and older adolescents. Duration of both illness and secondary amenorrhea was similar between groups. 
Relationships among BMI, estradiol level, percentage body fat, and percent median body weight (MBW) with ROM are shown in Figure 1. In longitudinal models, higher percentage body fat was associated with ROM (odds ratio [OR], 1.19; 95\% confidence interval [CI], $1.06,1.33 ; p<.01)$, while change in fat percentage was not. At 18 months, mean percentage body fat was $23.1 \%$ in subjects with ROM $(n=22)$ versus $18.1 \%$ in those without $(n=7$; Figure 1). Both BMI and change in BMI from baseline were associated with ROM (BMI: OR, 1.48; 95\% CI, 1.13,1.95; $p$ <.01 and change in BMI: OR, 1.53; 95\% CI, 1.09, 2.14; $p$ $=.02)$. At 18 months, subjects with ROM had a larger mean change in BMI $\left(2.2 \mathrm{~kg} / \mathrm{m}^{2}\right)$ than those without $\left(.90 \mathrm{~kg} / \mathrm{m}^{2}\right)$.

While estradiol level was associated with ROM (OR, 1.02; 95\% CI, 1.01, 1.03; $p=.01)$, having estradiol above $330 \mathrm{pg} / \mathrm{mL}$ was not $(\mathrm{p}=.08)$. Only the combination of estradiol $\geq 30$ $\mathrm{pg} / \mathrm{mL}$ and percent MBW >92\% was associated with ROM (OR, 2.49; 95\% CI, 1.09, 5.65; $p$ $=.03$ ). At 18 months, mean estradiol was $72.5 \pm 67.5 \mathrm{pg} / \mathrm{mL}$ in those with ROM and $19.9 \pm$ 11.3 in those without. Percent MBW alone predicted ROM (OR, 1.09; 95\% CI, 1.03, 1.16; $p$ $=.004)$, as did change in percent MBW (OR, 1.09; 95\% CI, 1.01, 1.18; $p=.02)$.

While leptin levels increased in both groups $(4.6 \pm 5.3 \mathrm{ng} / \mathrm{mL}$ at 18 months in those with $\mathrm{ROM}, \mathrm{n}=8 ; 3.5 \pm 3.1 \mathrm{ng} / \mathrm{mL}$ at 18 months in those without), neither leptin nor change therein was associated with ROM. Cortisol was not associated with ROM.

Although mean scores on mental health indicators decreased during study participation, no associations between ROM were seen.

\section{Discussion}

Weight, menstrual status, and mental health are important considerations when caring for patients with AN. Weight restoration remains a primary treatment aim.

Similar to what Golden et al. [1] demonstrated, weight gain coupled with estradiol $\geq 30$ $\mathrm{pg} / \mathrm{mL}$ was associated with ROM in the current sample. While estradiol $230 \mathrm{pg} / \mathrm{mL}$ alone was not significantly associated with ROM in this study, the relationship may have been seen with a larger sample. Our data also suggest that percentage body fat is associated with ROM, consistent with literature regarding menstrual dysfunction in athletes with low body fat $[9,10]$. Many adolescents with AN are assessed by DXA, given its accuracy measuring bone density, and the body composition analysis tool DXA also affords could aid clinicians in determining patient progress toward ROM.

The "ideal" percentage body fat for ROM likely varies among individuals as does "ideal" body weight. In a study of female athletes, subjects with amenorrhea had a mean percentage body fat of $23 \%$ versus $26.2 \%$ among ovulatory subjects [10]. Our subjects with ROM had closer to $23 \%$ body fat. The difference may be explained by physical activity. Only $54 \%$ of our subjects with ROM regularly exercised and for a shorter time than subjects in the study by Mallinson et al. Greater routine energy expenditure may require a higher percentage body fat for reproductive function. 
Given that leptin is chiefly obtained for research purposes, body composition assessments may be more feasible to follow longitudinally. We propose that with a larger sample size, leptin may have represented a significant predictor of ROM. Further research is needed to explore this hypothesis.

Clinicians often cite psychopathology and eating-disordered thinking as a cause of amenorrhea in patients who appear weight restored but remain amenorrheic. While important to overall health, improvement in mental well-being did not contribute to ROM in this sample.

Limitations include the small sample size and fact that the parent trial was not designed to examine associations with ROM. Measures of psychopathology were self-reported, which predisposes to bias in a clinical population prone to secrecy and denial. However, our data support previously published findings [1] and raise the possibility that percentage body fat is an additional clinical measure to consider in caring for adolescents with AN.

\section{Acknowledgments}

We thank the dedicated nursing and DXA staff of the Boston Children's Hospital Clinical and Translational Study Unit and our patients and their families.

Funding Sources: We gratefully acknowledge our funding; R01 HD043869, K23 HD060066, R01 AR060829, Department of Defense, US Army Bone Health and Military Readiness Program, and Harvard Catalyst, The Harvard Clinical and Translational Science Center (National Center for Research Resources and the National Center for Advancing Translational Sciences, National Institutes of Health Award 8UL1TR000170-05 and financial contributions from Harvard University and its affiliated academic health care centers). The content is solely the responsibility of the authors and does not necessarily represent the official views of Harvard Catalyst, Harvard University and its affiliated academic health care centers, or the National Institutes of Health.

\section{References}

1. Golden NH, Jacobsen MS, Sterling WM, Hertz S. Treatment goal weight in adolescents with anorexia nervosa: Use of BMI percentiles. Int J Eat Disord. 2008; 41:301-6. [PubMed: 18176951]

2. Munoz MT, Argent J. Anorexia nervosa in female adolescents: Endocrine and bone mineral density disturbances. Eur J Endocrinol. 2002; 147:275-86. [PubMed: 12213663]

3. Brown LM, Clegg DJ. Estrogen and leptin regulation of endocrinological features of anorexia nervosa. Neuropsychopharmacology. 2013; 38:237. [PubMed: 23147480]

4. Welt CK, Chan JL, Bullen J, et al. Recombinant human leptin in women with hypothalamic amenorrhea. N Engl J Med. 2004; 351:987-97. [PubMed: 15342807]

5. Gordon CM. Functional hypothalamic amenorrhea. N Engl J Med. 2010; 363:365-71. [PubMed: 20660404]

6. DiVasta AD, Feldman HA, Giancaterino C, et al. The effect of gonadal and adrenal steroid therapy on skeletal health in adolescents and young women with anorexia nervosa. Metabolism. 2012; 61:1010-20. [PubMed: 22257645]

7. Beck AT, Ward CH, Mendelson M, et al. An inventory for measuring depression. Arch Gen Psychiatry. 1961; 4:561-71. [PubMed: 13688369]

8. Garner DM, Olmsted MP, Bohr Y, Garfinkel PE. The eating attitudes test: Psychometric features and clinical correlates. Psychol Med. 1982; 12:871-8. [PubMed: 6961471]

9. Ackerman KE, Slysarz K, Guereca G, et al. Higher ghrelin and lower leptin secretion are associated with lower LH secretion in young amenorrheic athletes compared with eumenorrheic athletes and controls. Am J Physiol Endocrinol Metab. 2012; 302:E800-6. [PubMed: 22252944] 
10. Mallinson RJ, Williams NI, Hill BR, De Souza MJ. Body composition and reproductive function exert unique influences on indices of bone health in exercising women. Bone. 2013; 56:91-100. [PubMed: 23702387] 

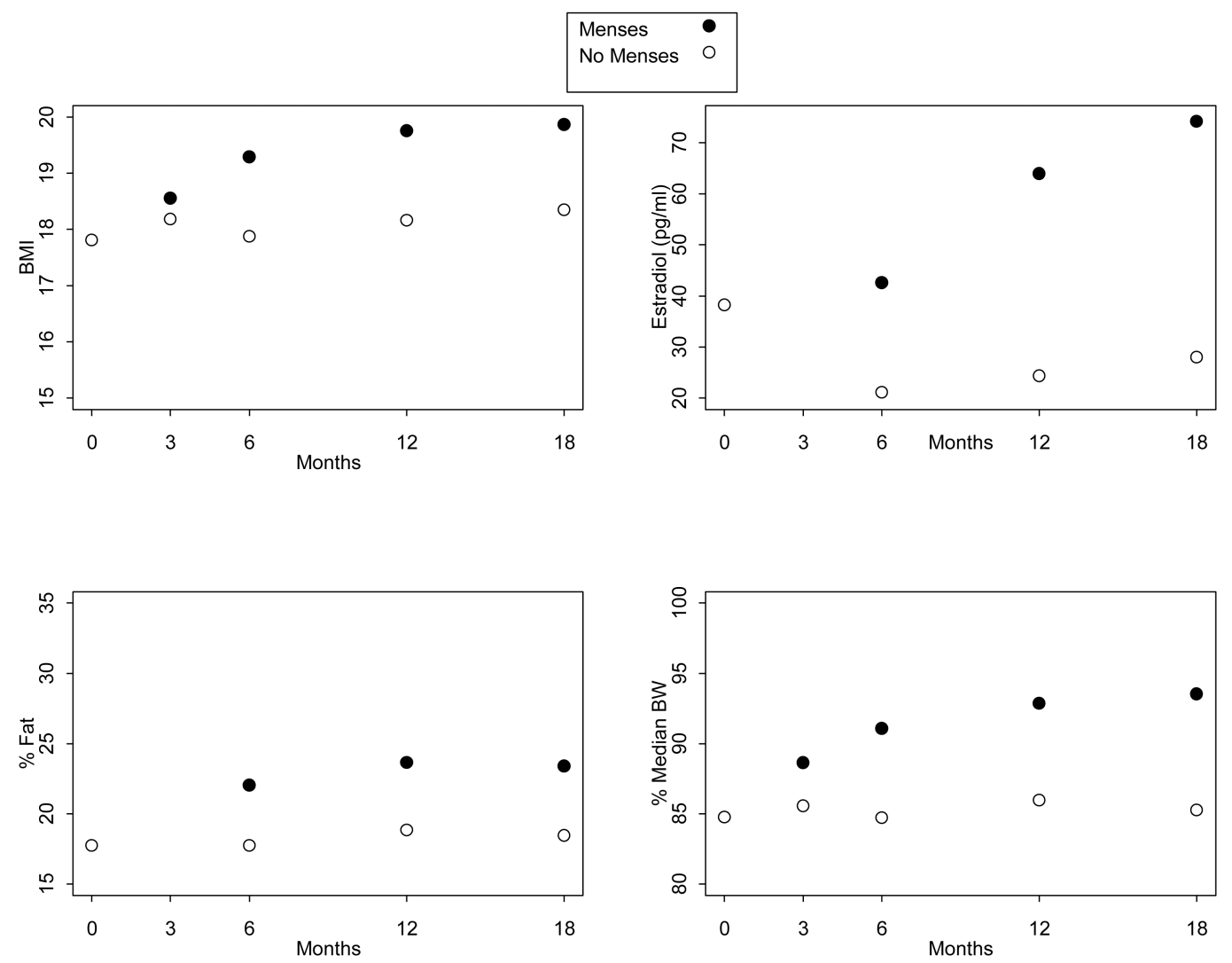

Figure 1.

Mean outcome measures (body mass index (BMI), estradiol, percentage fat, and percent median body weight) over time by whether or not menses had resumed. 
Table 1

Baseline characteristics overall and as a function of menstrual resumption during 18 months of follow-up

\begin{tabular}{lrrrr}
\hline & Overall, $\mathbf{n}=\mathbf{3 7}$ & No ROM, $\mathbf{n}=\mathbf{1 3}$ & $\mathbf{R O M}, \mathbf{n}=\mathbf{2 4}$ & $\boldsymbol{p}$ Value $^{\boldsymbol{a}}$ \\
\hline Age (years), mean (SD) & $18(2.8)$ & $18(1.7)$ & $18(3.1)$ & .38 \\
Race & & & & .47 \\
$\quad$ White & $32(86)$ & $10(77)$ & $22(92)$ & \\
$\quad$ Asian & $2(5)$ & $1(8)$ & $1(4)$ & \\
$\quad$ Other & $3(8)$ & $2(15)$ & $1(4)$ & \\
Non-Hispanic Ethnicity & $36(97)$ & $13(100)$ & $23(96)$ & 1.0 \\
Months since last period, mean (SD) & $20(12)$ & $25(23)$ & $18(29)$ & .13 \\
Months since AN diagnosis, mean (SD) & $19(26)$ & $20(14)$ & $18(29)$ & .70 \\
Regular exercise & $24(65)$ & $11(85)$ & $13(54)$ & .08 \\
Hours of exercise/week & $7(3)$ & $7(3)$ & $6(3)$ & .17 \\
BMI (kg/m $\left.{ }^{2}\right)$, mean (SD) & $17.8(1.5)$ & $18.1(1.7)$ & $17.7(1.4)$ & .61 \\
Percent body fat, mean (SD) & $17.8(5.1)$ & $17.3(5.5)$ & $18.0(5.0)$ & .05 \\
Cortisol $(\mu \mathrm{gg} / \mathrm{dL})$, mean (SD) & $15.9(7.6)$ & $16.5(8.3)$ & $15.6(7.3)$ & .06 \\
Estradiol (pg/mL), mean (SD) & $38.2(69.8)$ & $40.2(62.1)$ & $37.3(74.5)$ & .37 \\
Leptin (ng/mL), ${ }^{a}$ mean (SD) & $3.8(1.7)$ & $2.5(1.3)$ & $4.1(1.7)$ & $<.001$ \\
BDI, mean (SD) & $14.1(9.3)$ & $12.5(9.8)$ & $14.9(9.2)$ & .06 \\
EAT-26, mean (SD) & $24.3(17.5)$ & $24.9(18.3)$ & $24.0(17.4)$ & .83 \\
\hline
\end{tabular}

$p$ Value from Fisher's exact test for categorical variables and $t$ test for continuous variables.

$\mathrm{AN}=$ anorexia nervosa; BDI = Beck Depression Inventory-I; BMI = body mass index; EAT-26 = 26-item Eating Attitudes Test; M = month; ROM $=$ resumption of menses; $\mathrm{SD}=$ standard deviation.

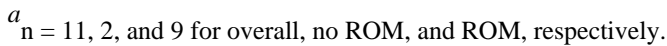

